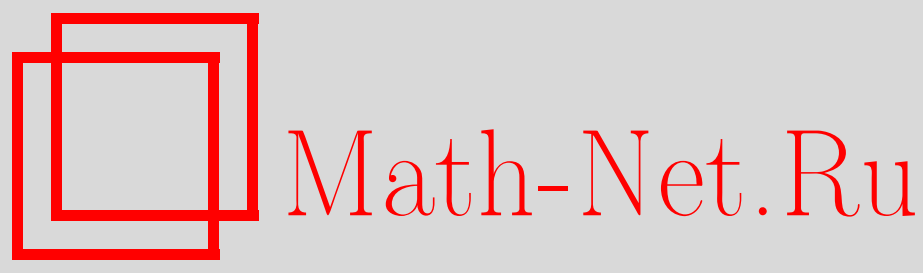

Е. А. Иванов, Суперсимметризация моделей Ландау, TMФ, 2008, том 154, номер 3, 409-423

DOI: https://doi.org/10.4213/tmf6179

Использование Общероссийского математического портала Math-Net.Ru подразумевает, что вы прочитали и согласны с пользовательским соглашением http://www . mathnet.ru/rus/agreement

Параметры загрузки:

IP: 54.172 .240 .79

26 апреля 2023 г., $15: 40: 21$

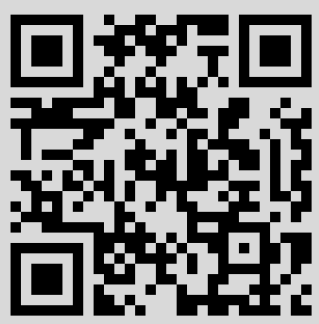




\section{СУПЕРСИММЕТРИЗАЦИЯ МОДЕЛЕЙ ЛАНДАУ}

Дан обзор последних работ, посвященных построению и изучению суперрасширений задачи Ландау о квантовой частице на плоскости в однородном магнитном поле, а также обобщения этой задачи на случай сферы $S^{2}$, сделанного Хэлдейном. Основное внимание уделено планарным суперсимметричным моделям Ландау, которые инвариантны относительно неоднородной супергруппы $I S U(1 \mid 1)$, контракции супергруппы $S U(2 \mid 1)$, и представляют собой минимальные суперрасширения исходной модели Ландау. Их общее существенное свойство - наличие скрытой динамической $\mathcal{N}=2$ суперсимметрии на мировой линии, которая присутствует как на классическом, так и на квантовом уровнях и наиболее естественно обнаруживается при переходе к новому инвариантному внутреннему произведению в пространстве квантовых состояний, что необходимо для обеспечения положительности всех норм. Для одной из планарных моделей - модели Ландау на суперплоскости - представлена формулировка вне массовой поверхности в терминах суперполей на мировой линии, позволяющая сделать $\mathcal{N}=2$ суперсимметрию явной.

Ключевые слова: суперсимметрия, суперполе, супергруппа, частица.

\section{1. ВВЕДЕНИЕ}

Модель Ландау [1] описывает заряженную частицу, движущуюся на плоскости под воздействием однородного магнитного поля, ортогонального к плоскости. Сферический вариант модели Ландау (модель Хэлдейна [2]) описывает заряженную частицу на сфере $S^{2} \sim S U(2) / U(1)$ в поле монополя Дирака, помещенного в центр. Эти модели имеют много приложений, в частности, в квантовом эффекте Холла $(\mathrm{K} \ni \mathrm{X})[3]$.

Суперсимметричные расширения модели Ландау, о которых пойдет речь в настоящей работе, представляют собой модели нерелятивистских суперчастиц, движущихся на супермногообразиях. Изучение таких моделей может прояснить вопрос о возможных проявлениях суперсимметрии в различных версиях КЭХ (включая так называемый спиновый $\mathrm{K} Э \mathrm{X}$ ) и других реалистических моделях конденсированных сред. В математическом аспекте модель Ландау и ее суперрасширения тесно связаны с некоммутативной (супер)геометрией: после квантования в них возникает взаимно однозначное соответствие между нижними уровнями Ландау (УЛ) и

* Объединенный институт ядерных исследований, Дубна, Московская обл., Россия. E-mail: eivanov@theor.jinr.ru 
не(анти)коммутативными (супер)многообразиями, которые представлены соответствующими координатными операторами.

Задачи Ландау на суперсфере $S U(2 \mid 1) / U(1 \mid 1)$ размерности $(2 \mid 2)$ и суперфлаге $S U(2 \mid 1) /[U(1) \times U(1)]$ размерности $(2 \mid 4)$, т.е. минимальные суперрасширения модели Хэлдейна на $S^{2}$, рассмотрены в работах [4], [5] (см. также работу [6]). Для лучшего понимания общих особенностей суперсимметричных моделей Ландау оказалось полезным изучить планарные пределы таких суперобобщений. Планарные модели должны получаться из своих криволинейных прототипов в пределе большого радиуса сферы $S^{2}$ (пределе контракции). Такие модели были построены и исследованы в работах [7], [8], а также [9]. Модель, полученная из $S U(2 \mid 1) / U(1 \mid 1)$-модели, называется моделью Ландау на суперплоскости, а модель, возникающая из $S U(2 \mid 1) /[U(1) \times U(1)]$-модели, носит название модель Ландау на планарном суперфлаге.

Данная работа представляет собой обзор суперсимметричных моделей Ландау [4], [5], [7], [8] с упором на их планарные варианты и присутствие в последних скрытой $\mathcal{N}=2$ суперсимметрии на мировой линии, что, по-видимому, является общим свойством планарных моделей [8], [9]. Для модели Ландау на суперплоскости в качестве существенно нового результата развит $\mathcal{N}=2$ суперполевой формализм, позволяющий сделать явной $\mathcal{N}=2$ суперсимметрию на мировой линии. Кроме того, обсуждается проблема присутствия состояний с отрицательной нормой [7] в квантовых планарных моделях Ландау; показано, что во всех случаях эту трудность можно преодолеть за счет введения нетривиальной метрики на пространстве состояний в духе работ [10], [11].

\section{2. ПРЕАМБУЛА: БОЗОННЫЕ МОДЕЛИ ЛАНДАУ}

\section{1. Бозонные модели Ландау как $d=1$ сигма-модели ВЗВ-типа.}

2.1.1. Определения. Стандартная планарная модель Ландау описывается следующим лагранжианом:

$$
L_{\mathrm{b}}=|\dot{z}|^{2}-i \kappa(\dot{z} \bar{z}-\dot{\bar{z}} z)=|\dot{z}|^{2}+\left(A_{z} \dot{z}+A_{\bar{z}} \dot{\bar{z}}\right)
$$

Здесь $z(t), \bar{z}(t)$ - комплексные координаты двумерной евклидовой плоскости, $2 \kappa-$ напряженность внешнего однородного магнитного поля,

$$
A_{z}=-i \kappa \bar{z}, \quad A_{\bar{z}}=i \kappa z, \quad \partial_{\bar{z}} A_{z}-\partial_{z} A_{\bar{z}}=-2 i \kappa .
$$

Второй член в (2.1) - простейший пример члена Весса-Зумино (В3).

Соответствующий канонический гамильтониан представляется в виде

$$
H=\frac{1}{2}\left(a^{\dagger} a+a a^{\dagger}\right)=a^{\dagger} a+\kappa,
$$

где

$$
a=i\left(\partial_{\bar{z}}+\kappa z\right), \quad a^{\dagger} i\left(\partial_{z}-\kappa \bar{z}\right), \quad\left[a, a^{\dagger}\right]=2 \kappa .
$$


Он коммутирует со следующими операторами, которые, таким образом, определяют инвариантности данной теории (см., например, работу [12]):

$$
\begin{gathered}
P_{z}=-i\left(\partial_{z}+\kappa \bar{z}\right), \quad P_{\bar{z}}=-i\left(\partial_{\bar{z}}-\kappa z\right), \\
{\left[P_{z}, P_{\bar{z}}\right]=2 \kappa, \quad F_{\mathrm{b}}=z \partial_{z}-\bar{z} \partial_{\bar{z}},} \\
{\left[H, P_{z}\right]=\left[H, P_{\bar{z}}\right]=\left[H, F_{\mathrm{b}}\right]=0 .}
\end{gathered}
$$

Операторы $P_{z}, P_{\bar{z}}$ и $F_{\mathrm{b}}$ генерируют соответственно “магнитные трансляции" и вращения в $2 D$-мерном "пространстве отображения". Эти операторы суть квантовые аналоги нетёровских зарядов, связанных с инвариантностью относительно трансляций $z^{\prime}=z+a, \bar{z}^{\prime}=\bar{z}+\bar{a}$ и $U(1)$-вращений $z^{\prime}=e^{i \alpha} z, \bar{z}^{\prime}=e^{-i \alpha} \bar{z}$.

2.1.2. Волновые функции. Волновая функция $\Psi_{0}$, соответствующая низшему УЛ, $H \Psi_{(0)}=\kappa \Psi_{(0)}$, определяется уравнением

$$
a \Psi_{(0)}(z, \bar{z})=0 \quad \Longleftrightarrow \quad\left(\partial_{\bar{z}}+\kappa z\right) \Psi_{(0)}=0 \Longrightarrow \Psi_{(0)}=e^{-\kappa|z|^{2}} \psi_{(0)}(z),
$$

т.е. сводится к голоморфной функции.

Волновая функция, отвечающая $n$-му УЛ, строится как

$$
\Psi_{(n)}(z, \bar{z})=\left[i\left(\partial_{z}-\kappa \bar{z}\right)\right]^{n} e^{-\kappa|z|^{2}} \psi_{(n)}(z), \quad H \Psi_{(n)}=\kappa(2 n+1) \Psi_{(n)},
$$

т.е. также выражается через голоморфную функцию. Каждый УЛ бесконечно вырожден вследствие $\left(P_{z}, P_{\bar{z}}\right)$-инвариантности. На каждом УЛ волновые функции образуют бесконечномерные представления этой группы с базисом $z^{m}, m>0$ [12].

Инвариантная норма определяется как интеграл

$$
\left\|\Psi_{(n)}\right\|^{2}=\int d z d \bar{z} \bar{\Psi}_{(n)}(z, \bar{z}) \Psi_{(n)}(z, \bar{z}) \sim \int d z d \bar{z} e^{-2 \kappa|z|^{2}} \bar{\psi}_{(n)}(\bar{z}) \psi_{(n)}(z)<\infty .
$$

Он сходится для любого монома $\psi_{(n)}(z) \sim z^{m}$.

2.1.3. Планарная модель Ландау как ВЗВ-модель. Будем считать $2 \kappa$ в $\left[P_{z}, P_{\bar{z}}\right]=$ $2 \kappa$ независимым генератором ("центральным зарядом") и построим нелинейную реализацию этой неабелевой группы магнитных трансляций в фактор-пространстве по одномерной подгруппе, порождаемой $2 \kappa$. Выбирая экспоненциальную параметризацию для соответствующих элементов фактор-пространства, получаем

$$
\begin{gathered}
g(z, \bar{z})=e^{i\left(z P_{z}+\bar{z} P_{\bar{z}}\right)}, \quad g^{-1} d g=i \omega_{z} P_{z}+i \omega_{\bar{z}} P_{\bar{z}}+i \omega_{\kappa} 2 \kappa, \\
\omega_{z}=d z, \quad \omega_{\bar{z}}=d \bar{z}, \quad \omega_{\kappa}=\frac{1}{2 i}(z d \bar{z}-\bar{z} d z) .
\end{gathered}
$$

Мы видим, что ВЗ-член в $L_{\mathrm{b}}$ есть не что иное, как 1-форма Картана, связанная с генератором $2 \kappa$. В этой геометрической постановке операторы рождения и уничтожения $a^{\dagger}$ и $a$ возникают как ковариантные производные:

$$
\nabla_{z}=\partial_{z}-\kappa \bar{z}, \quad \nabla_{\bar{z}}=\partial_{\bar{z}}+\kappa z
$$

в то время как волновая функция, отвечающая низшему УЛ, определяется ковариантным условием Коши-Римана:

$$
\nabla_{\bar{z}} \Psi_{(0)}=0, \quad \Psi_{(n)}^{\prime}\left(z^{\prime}, \bar{z}^{\prime}\right)=e^{-\kappa(a \bar{z}-\bar{a} z)} \Psi_{(n)}(z, \bar{z}), \quad z^{\prime}=z+a, \quad \bar{z}^{\prime}=\bar{z}+\bar{a} .
$$


2.1.4. Обобщение на $S^{2}$. Аналог планарного лагранжиана Ландау $L_{\mathrm{b}}$ на сфере $S^{2}$ (т.е. лагранжиан модели Хэлдейна) записывается в виде

$$
\widetilde{L}_{\mathrm{b}}=\frac{1}{\left(1+r^{2}|z|^{2}\right)^{2}}|\dot{z}|^{2}-i s \frac{1}{1+r^{2}|z|^{2}}(\dot{z} \bar{z}-\dot{\bar{z}} z) .
$$

Первый член здесь - это $(d=1)$-проекция квадрата инвариантного интервала на $S^{2}$, второй член - $(d=1)$-член В3 на фактор-пространстве $S^{2} \sim S U(2) / U(1), r-$ "обратный" радиус $S^{2}$.

После квантования унитарные волновые функции на каждом уровне образуют конечномерные неприводимые представления группы $S U(2)$ со "спинами" $s, s+1 / 2$, $s+1, \ldots$. Таким образом, каждый УЛ имеет конечное вырождение (в этом состоит важное отличие от планарного случая, и связано оно с тем фактом, что $S U(2)$ является компактной группой, в то время как ее контракция, группа магнитных трансляций, не компактна). Волновая функция низшего УЛ определяется ковариантным условием аналитичности на $S^{2}$ :

$$
\nabla_{\bar{z}} \Psi_{(0)}=0, \quad \nabla_{\bar{z}}=\left(1+r^{2}|z|^{2}\right) \partial_{\bar{z}}+U(1) \text {-связность. }
$$

Можно показать, что эта волновая функция сводится к голоморфной функции $z$, компоненты которой образуют неприводимый $S U(2)$-мультиплет со спином $s$. Волновые функции, отвечающие высшим УЛ, строятся во многом подобно планарному случаю и выражаются через голоморфные функции, описывающие неприводимые $S U(2)$-мультиплеты со спинами $s+1 / 2, s+1, \ldots$. В пределе $r \rightarrow 0$ воспроизводится планарная модель Ландау.

2.2. Низший УЛ и пространственная некоммутативность. Вернемся к случаю планарной модели Ландау. Разность энергий низшего и первого УЛ есть $E_{1}-E_{0}=\kappa$, и она стремится к бесконечности с ростом $\kappa$. В этом пределе выживает только низший УЛ, который описывается ВЗ-членом. Условие аналитичности $\nabla_{\bar{z}} \Psi_{(0)}=0$ теперь возникает как квантовая версия соответствующей связи в фазовом пространстве. Стандартный оператор координаты $Z=z$ не коммутирует с этой связью и поэтому должен быть подходящим образом модифицирован:

$$
Z=z-\frac{1}{\kappa} \partial_{\bar{z}}=\frac{1}{i \kappa} P_{\bar{z}}, \quad \bar{Z}=\frac{i}{\kappa} P_{z}, \quad[\bar{Z}, Z]=\frac{2}{\kappa} .
$$

Новые операторы $Z, \bar{Z}$ параметризуют некоммутативную плоскость.

Подобная ситуация имеет место и в $S^{2}$-случае: в пределе низшего УЛ выживает только ВЗ-член, и соответствующие операторы координат, коммутирующие с гамильтоновыми связями, параметризуют некоммутативную ("размытую") версию $S^{2}[13]$.

\section{3. СУПЕРСИММЕТРИЗАЦИЯ МОДЕЛИ ЛАНДАУ: ПРОСТОЙ ПРИМЕР}

Под суперсимметричными моделями Ландау мы будем понимать квантово-механические модели заряженной частицы на однородном суперпространстве такие, что в бозонном пределе они сводятся либо к исходной модели Ландау для заряженной частицы, движущейся на плоскости в однородном магнитном поле, либо к ее сферической версии, разработанной Хэлдейном. 


\section{1. Суперсимметрия мировой линии и суперсимметрия в пространстве} отображения. Один из путей суперсимметризации моделей Ландау состоит в том, что вводится суперсимметрия на мировой линии

$$
t \quad \Rightarrow \quad(t, \theta, \bar{\theta}), \quad z, \bar{z} \quad \Rightarrow \quad \mathcal{Z}(t, \theta, \bar{\theta}), \overline{\mathcal{Z}}(t, \theta, \bar{\theta}),
$$

$z, \bar{z} \Rightarrow(z, \bar{z}, \psi, \bar{\psi}, \ldots)$ - супермультиплет на мировой линии. Здесь $\mathcal{Z}$ - некоторые $(d=1)$-суперполя. Соответствующие модели отвечают той или иной версии суперсимметричной квантовой механики [14] (см., например, работу [15]). В этом варианте суперсимметризации фермионные поля не имеют непосредственной геометрической интерпретации в отличие от бозонных полей $z$, $\bar{z}$, которые, как мы видели, являются параметрами фактор-пространства.

Другой способ суперрасширения моделей Ландау состоит во введении суперсимметрии в пространстве отображения. Простейшая возможность - расширить группу магнитных трансляций до супергруппы, включающей генераторы “магнитных супертрансляций” $\Pi_{\zeta}, \Pi_{\bar{\zeta}}$ и фермионный аналог $U(1)$-генератора $F_{\mathrm{b}}$ :

групповое многообразие: $(z, \bar{z}) \Rightarrow$ супергрупповое многообразие $:(z, \bar{z}, \zeta, \bar{\zeta})$,

$$
\begin{gathered}
\left(P_{z}, P_{\bar{z}}, F_{\mathrm{b}}, \kappa\right) \Rightarrow \quad\left(P_{z}, P_{\bar{z}}, \Pi_{\zeta}, \Pi_{\bar{\zeta}}, F_{\mathrm{b}}, F_{\mathrm{f}}, \kappa, \ldots\right), \\
\Pi_{\zeta}=\partial_{\zeta}+\kappa \bar{\zeta}, \quad \Pi_{\bar{\zeta}}=\partial_{\bar{\zeta}}+\kappa \zeta, \quad F_{\mathrm{f}}=\zeta \partial_{\zeta}-\bar{\zeta} \partial_{\bar{\zeta}}, \quad\left\{\Pi_{\zeta}, \Pi_{\bar{\zeta}}\right\}=2 \kappa .
\end{gathered}
$$

В этом случае фермионные поля имеют ясный геометрический смысл: это грассмановы координаты, расширяющие двумерную плоскость до (2|2)-мерной суперплоскости.

Примечательно, что две планарные суперсимметричные модели Ландау, построенные с привлечением второго подхода (см. разделы 4 и 5), вдобавок обладают еще и скрытой $\mathcal{N}=2$ суперсимметрией на мировой линии.

3.2. “Фермионная модель Ландау”. Прежде чем перейти к обсуждению суперсимметризаций моделей Ландау, стоит рассмотреть упрощенную "фермионную модель Ландау", в которой бозонные $2 D$-координаты $z, \bar{z}$ заменены фермионными $\zeta, \bar{\zeta}$.

Соответствующие лагранжиан и гамильтониан записываются в виде

$$
\begin{aligned}
L_{\mathrm{f}} & =\dot{\zeta} \dot{\bar{\zeta}}-i \kappa(\dot{\zeta} \bar{\zeta}+\dot{\bar{\zeta}} \zeta), \quad H_{\mathrm{f}}=\frac{1}{2}\left[\alpha, \alpha^{\dagger}\right]=-\alpha^{\dagger} \alpha-\kappa, \\
\alpha & =\partial_{\bar{\zeta}}-\kappa \zeta, \quad \alpha^{\dagger}=\partial_{\zeta}-\kappa \bar{\zeta}, \quad\left\{\alpha, \alpha^{\dagger}\right\}=-2 \kappa .
\end{aligned}
$$

Инвариантности порождаются генераторами $\Pi_{\zeta}, \Pi_{\bar{\zeta}}$ и $F_{\mathrm{f}}$, определенными в $(3.2)$,

$$
\left[H_{\mathrm{f}}, \Pi_{\zeta}\right]=\left[H_{\mathrm{f}}, \Pi_{\bar{\zeta}}\right]=\left[H_{\mathrm{f}}, F_{\mathrm{f}}\right]=0 .
$$

Квантовое "гильбертово пространство" модели включает основное состояние и единственное возбужденное состояние:

$$
\begin{gathered}
\psi^{(0)}=e^{-\kappa \zeta \bar{\zeta}} \psi_{0}(\zeta), \quad \psi^{(1)}=e^{\kappa \zeta \bar{\zeta}} \psi_{1}(\bar{\zeta}), \quad \alpha \psi^{(0)}=\alpha^{\dagger} \psi^{(1)}=0, \\
\psi_{0}=A_{0}+\zeta B_{0}, \quad \psi_{1}=A_{1}+\bar{\zeta} B_{1} .
\end{gathered}
$$


Пары $\left(A_{0}, B_{0}\right),\left(A_{1}, B_{1}\right)$ образуют два неприводимых мультиплета группы магнитных супертрансляций с энергиями $-\kappa$ и $\kappa$.

Уже в этом простейшем примере мы сталкиваемся с проблемой, которая присутствует также и в суперрасширениях модели Ландау, рассмотренных в следующих разделах. Это появление духов и необходимость их "изгнания".

При естественном инвариантном выборе внутреннего произведения,

$$
\langle\phi \mid \psi\rangle=\int d \zeta d \bar{\zeta} \overline{\phi(\zeta, \bar{\zeta})} \psi(\zeta, \bar{\zeta})
$$

получаем, что

$$
\begin{aligned}
\left\langle\psi^{(0)} \mid \psi^{(1)}\right\rangle & =0, \\
\left\langle\psi^{(0)} \mid \psi^{(0)}\right\rangle & =2 \kappa \bar{A}_{0} A_{0}+\bar{B}_{0} B_{0}, \\
\left\langle\psi^{(1)} \mid \psi^{(1)}\right\rangle & =-2 \kappa \bar{A}_{1} A_{1}-\bar{B}_{1} B_{1} .
\end{aligned}
$$

Состояния $A_{1}, B_{1}$ имеют отрицательную норму, т.е. они представляют собой духи ${ }^{1)}$. Как обычно, присутствие духов должно рассматриваться как нежелательное свойство, поскольку оно может привести к нарушению унитарности ${ }^{2)}$. В данном случае эту трудность можно преодолеть путем введения нетривиальной метрики на "гильбертовом пространстве":

$$
\langle\langle\phi \mid \psi\rangle\rangle:=\langle G \phi \mid \psi\rangle, \quad G\left(\psi^{(0)}+\psi^{(1)}\right)=\psi^{(0)}-\psi^{(1)}, \quad G=-\kappa^{-1} H_{\mathrm{f}} .
$$

Характерные черты этой процедуры сводятся к следующим.

1. Генераторы симметрий $\Pi_{\zeta}, \Pi_{\bar{\zeta}}$ и $F_{\mathrm{f}}$ коммутируют с метрикой $G$, поэтому новое внутреннее произведение остается инвариантным. Однако свойства эрмитова сопряжения операторов, которые не коммутируют с $G$, изменяются, например

$$
\alpha^{\ddagger}=-\alpha^{\dagger} \Rightarrow H_{\mathrm{f}}=\alpha^{\ddagger} \alpha-\kappa, \quad\left\{\alpha, \alpha^{\ddagger}\right\}=2 \kappa .
$$

2. Относительно нового произведения импульс, канонически сопряженный координате, оказывается также и эрмитово сопряженным той же координате:

$$
\zeta^{\ddagger}=\frac{1}{\kappa} \partial_{\zeta}, \quad(\bar{\zeta})^{\ddagger}=\frac{1}{\kappa} \partial_{\bar{\zeta}}, \quad\left(\partial_{\zeta}\right)^{\ddagger}=\kappa \zeta, \quad\left(\partial_{\bar{\zeta}}\right)^{\ddagger}=\kappa \bar{\zeta} .
$$

На этом мы закончим обсуждение упрощенной модели.

\section{4. МОДЕЛЬ ЛАНДАУ НА СУПЕРПЛОСКОСТИ}

4.1. Лагранжиан, гамильтониан и симметрии. Модель Ландау на суперплоскости может рассматриваться как "гибрид" бозонной и фермионной моделей Ландау. Она описывается следующим лагранжианом:

$$
L=L_{\mathrm{f}}+L_{\mathrm{b}}=|\dot{z}|^{2}+\dot{\zeta} \dot{\bar{\zeta}}-i \kappa(\dot{z} \bar{z}-\dot{\bar{z}} z+\dot{\zeta} \bar{\zeta}+\dot{\bar{\zeta}} \zeta)
$$

\footnotetext{
1) Появление духов в $(d=1)$-суперсимметричных моделях с кинетическими членами второго порядка для фермионов было отмечено в работе [16].

2) См., однако, работу [17].
} 
Соответствующий квантовый гамильтониан имеет вид

$$
H=a^{\dagger} a-\alpha^{\dagger} \alpha=\partial_{\bar{\zeta}} \partial_{\zeta}-\partial_{z} \partial_{\bar{z}}+\kappa\left(\bar{z} \partial_{\bar{z}}+\bar{\zeta} \partial_{\bar{\zeta}}-z \partial_{z}-\zeta \partial_{\zeta}\right)+\kappa^{2}(z \bar{z}+\zeta \bar{\zeta}) .
$$

Полный набор симметрий помимо тех, что порождены генераторами $P_{z}, P_{\bar{z}}$ и $\Pi_{\zeta}$, $\Pi_{\bar{\zeta}}$, включает также новые симметрии с генераторами

$$
\begin{gathered}
Q=z \partial_{\zeta}-\bar{\zeta} \partial_{\bar{z}}, \quad Q^{\dagger}=\bar{z} \partial_{\bar{\zeta}}+\zeta \partial_{z}, \quad C=F_{\mathrm{b}}+F_{\mathrm{f}}=z \partial_{z}+\zeta \partial_{\zeta}-\bar{z} \partial_{\bar{z}}-\bar{\zeta} \partial_{\bar{\zeta}} \\
{[H, Q]=\left[H, Q^{\dagger}\right]=[H, C]=0}
\end{gathered}
$$

Эти генераторы порождают супералгебру $I S U(1 \mid 1)$, являющуюся контракцией $S U(2 \mid 1)$ :

$$
\left\{Q, Q^{\dagger}\right\}=C, \quad[C, Q]=\left[C, Q^{\dagger}\right]=0, \quad\left[Q, P_{z}\right]=i \Pi_{\zeta}, \quad\left\{Q^{\dagger}, \Pi_{\zeta}\right\}=i P_{z} .
$$

4.2. Состояния и вырождение. По определению волновая функция низшего УЛ $\psi^{(0)}$ обращается в нуль под действием как бозонных, так и фермионных операторов уничтожения $a$ и $\alpha$ :

$$
\begin{aligned}
\left(\partial_{\bar{z}}+\kappa z\right) \psi^{(0)} & =\left(\partial_{\bar{\zeta}}-\kappa \zeta\right) \psi^{(0)}=0 \Rightarrow \\
\Rightarrow \psi^{(0)} & =e^{-\kappa K_{2}} \psi_{\text {an }}^{(0)}(z, \zeta), \quad K_{2}=|z|^{2}+\zeta \bar{\zeta}, \quad H \psi^{(0)}=0
\end{aligned}
$$

т.е. она имеет дополнительное двукратное вырождение, $\psi_{\text {an }}^{(0)}(z, \zeta)=A^{(0)}(z)+$ $\zeta B^{(0)}(z)$.

Гильбертово пространство для $N$-го УЛ определяется волновой функцией

$$
\begin{gathered}
\psi^{(N)} \sim\left(a^{\dagger}\right)^{N} e^{-\kappa K_{2}} \psi_{+}^{(N)}(z, \zeta)+\left(a^{\dagger}\right)^{N-1} \alpha^{\dagger} e^{-\kappa K_{2}} \psi_{-}^{(N)}(z, \zeta), \\
H \psi^{(N)}=2 \kappa N \psi^{(N)},
\end{gathered}
$$

где $\psi_{ \pm}^{(N)}(z, \zeta)=A_{ \pm}^{(N)}(z)+\zeta B_{ \pm}^{(N)}(z)$. Каждый УЛ с $N>0$ четырехкратно вырожден.

Естественное $I S U(1 \mid 1)$-инвариантное внутреннее произведение

$$
\langle\phi \mid \psi\rangle=\int d \mu \overline{\phi(z, \bar{z} ; \zeta, \bar{\zeta})} \psi(z, \bar{z} ; \zeta, \bar{\zeta}), \quad d \mu=d z d \bar{z} d \zeta d \bar{\zeta}
$$

приводит к отрицательным нормам для некоторых состояний, как и в упрощенной фермионной модели. Однако все нормы можно сделать положительными, вводя ту же самую метрику на гильбертовом пространстве:

$$
G=-\kappa^{-1} H_{\mathrm{f}}=\frac{1}{\kappa}\left[\partial_{\zeta} \partial_{\bar{\zeta}}+\kappa^{2} \bar{\zeta} \zeta+\kappa\left(\zeta \partial_{\zeta}-\bar{\zeta} \partial_{\bar{\zeta}}\right)\right]
$$

Метрика $G$ коммутирует с генераторами всех симметрий за исключением $Q, Q^{\dagger}$. Следовательно, по отношению к новому произведению $\langle G \phi \mid \psi\rangle$ оператор, сопряженный к $Q$, не совпадает с $Q^{\dagger}$. Новый сопряженный оператор $Q^{\ddagger}$ легко вычисляется:

$$
Q^{\ddagger}=Q^{\dagger}-\frac{i}{\kappa} S, \quad S=i\left(\partial_{z} \partial_{\bar{\zeta}}+\kappa^{2} \bar{z} \zeta-\kappa \bar{z} \partial_{\bar{\zeta}}-\kappa \zeta \partial_{z}\right) .
$$

Поскольку оба оператора $Q^{\dagger}$ и $Q^{\ddagger}$ коммутируют с полным гамильтонианом $H$ (так как $[H, G]=0)$, их разность $S$ также коммутирует с ним, $[H, S]=\left[H, S^{\ddagger}\right]=0$, и, следовательно, порождает новую (скрытую) симметрию модели. 
4.3. Скрытая $\mathcal{N}=2$ суперсимметрия мировой линии. Операторы $S, S^{\ddagger}$ выражаются в виде

$$
S=a^{\dagger} \alpha, \quad S^{\ddagger}=a \alpha^{\ddagger}
$$

и, как легко проверить, генерируют $\mathcal{N}=2$ суперсимметрию на мировой линии:

$$
[H, S]=\left[H, S^{\ddagger}\right]=0, \quad\left\{S, S^{\ddagger}\right\}=2 \kappa H, \quad\{S, S\}=0=\left\{S^{\ddagger}, S^{\ddagger}\right\} .
$$

Другими словами, $(2 \kappa)^{-1 / 2} S,(2 \kappa)^{-1 / 2} S^{\ddagger}$ и $H$ образуют $\mathcal{N}=2, d=1$ супералгебру Пуанкаре.

Генераторы $S, S^{\ddagger}$ аннигилируют основное состояние, соответствующее низшему УЛ:

$$
S \psi^{(0)}=S^{\ddagger} \psi^{(0)}=0,
$$

поэтому основное состояние является синглетом $\mathcal{N}=2$ суперсимметрии. Следовательно, $\mathcal{N}=2$ суперсимметрия не нарушена, и волновые функции, отвечающие высшим УЛ, образуют ее неприводимые мультиплеты. Каждое такое состояние состоит из двух неприводимых мультиплетов супергруппы $I S U(1 \mid 1)$, чем объясняется четырехкратное вырождение УЛ с $N>0$. Интересно, что для $\mathcal{N}=2$ суперсимметрии существует следующий конечномерный аналог модельно-независимого представления Сугавары в терминах $I S U(1 \mid 1)$-зарядов:

$$
S=2 i \kappa Q^{\ddagger}+P \Pi^{\ddagger}, \quad S^{\ddagger}=-2 i \kappa Q+P^{\ddagger} \Pi, \quad H=P^{\ddagger} P+\Pi^{\ddagger} \Pi-2 \kappa C,
$$

т.е. $S, S^{\ddagger}, H$ принадлежат обертывающей алгебре супералгебры $I S U(1 \mid 1)$.

На классическом уровне скрытая суперсимметрия мировой линии реализуется следующими преобразованиями полей $z, \zeta$ и сопряженных к ним:

$$
\delta z=\epsilon \dot{\zeta}, \quad \delta \zeta=-\dot{z} \bar{\epsilon}
$$

Как и должно быть, на массовой поверхности они замыкаются на производную полей по времени, если учесть уравнения движения $\ddot{z}=2 i \kappa \dot{z}, \ddot{\zeta}=2 i \kappa \dot{\zeta}$. $\mathcal{N}=2$ суперсимметрия существует только при $\kappa \neq 0$, т.е. она представляет собой род динамической суперсимметрии.

В разделе 6 будет показано, как воспроизвести эту реализацию исходя из явно $\mathcal{N}=2$ суперсимметричного суперполевого подхода вне массовой оболочки.

\section{5. МОДЕЛЬ ПЛАНАРНОГО СУПЕРФЛАГА}

В этом разделе мы рассмотрим основные черты еще одной $I S U(1 \mid 1)$-инвариантной модели, обобщающей модель Ландау: модели Ландау на планарном суперфлаге [7]. 


\section{1. Классическая и квантовая структуры модели.}

5.1.1. Определения. Модель Ландау на суперфлаге [5] описывает заряженную частицу на фактор-суперпространстве $S U(2 \mid 1) /[U(1) \times U(1)]$. Один из двух ВЗ-членов на подгруппе $U(1) \times U(1)$ есть лоренцев член взаимодействия с однородным магнитным полем напряженности $2 \kappa$ на $S^{2}$. Второй ВЗ-член является чисто фермионным, с постоянным коэффициентом $M$.

В планарном пределе, когда $S U(2 \mid 1)$ переходит в $I S U(1 \mid 1)$ и $S^{2}$ - в евклидову двумерную плоскость, действие, полученное в работе [5], переходит в следующее действие [7]:

$$
L=(1+\bar{\xi} \xi)|\dot{z}|^{2}+(\bar{\xi} \dot{\bar{z}} \dot{\zeta}-\xi \dot{\bar{z}} \dot{\bar{\zeta}})+\bar{\xi} \xi \dot{\zeta} \dot{\bar{\zeta}}-i \kappa(\dot{z} \bar{z}-\dot{\bar{z}} z+\dot{\zeta} \bar{\zeta}+\dot{\bar{\zeta}} \zeta)+i M(\bar{\xi} \dot{\xi}+\xi \dot{\bar{\xi}})
$$

Основное отличие этой модели от модели Ландау на суперплоскости состоит в том, что в (5.1) фигурирует дополнительная фермионная переменная $\xi(t), \bar{\xi}(t)$. Ее можно интерпретировать как поле Намбу-Голдстоуна, связанное с $I S U(1 \mid 1)$-генераторами $Q, Q^{\dagger}$. Благодаря этой дополнительной переменной удается построить второй ВЗ-член и одновременно избежать появления нестандартного кинетического члена второго порядка для $\zeta, \bar{\zeta}$. Несмотря на эти привлекательные свойства в квантовой теории при естественном определении внутреннего произведения все еще присутствуют отрицательные нормы.

5.1.2. Квантование. В теории имеются связи на фазовом пространстве (из-за фермионных членов первого порядка в (5.1)). Решая эти связи, можно найти общую структуру волновой функции:

$$
\Psi=K_{1}^{M} e^{-\kappa K_{2}} \Psi_{\mathrm{ch}}\left(z, \bar{z}_{\mathrm{sh}}, \zeta, \xi\right), \quad K_{1}=1+\bar{\xi} \xi, \quad \bar{z}_{\mathrm{sh}}=\bar{z}-\xi \bar{\zeta} .
$$

Операторный гамильтониан в применении к этим “физическим" волновым функциям записывается в виде

$$
\begin{array}{cl}
H=\hat{a}^{\dagger} \hat{a}, & {\left[\hat{a}, \hat{a}^{\dagger}\right]=2 \kappa,} \\
\hat{a}=i \sqrt{K_{1}}\left(\partial_{\bar{z}}+\kappa z_{\mathrm{sh}}-\bar{\xi} \partial_{\bar{\zeta}}\right), & \hat{a}^{\dagger}=i \sqrt{K_{1}}\left(\partial_{z}-\kappa \bar{z}_{\mathrm{sh}}-\xi \partial_{\zeta}\right) .
\end{array}
$$

На $N$-м УЛ физическая киральная волновая функция имеет специальный вид: она выражается через аналитическую функцию от $(z, \zeta, \xi)$ согласно формуле

$$
\Psi_{\mathrm{ch}}^{(N)}=\widetilde{\nabla}_{z}^{N} \Psi_{\mathrm{an}}^{(N)}(z, \zeta, \xi), \quad \widetilde{\nabla}_{z}=\partial_{z}-2 \kappa \bar{z}_{\mathrm{sh}}-\xi \partial_{\zeta}, \quad H \Psi_{\mathrm{ch}}^{(N)}=2 \kappa N \Psi_{\mathrm{ch}}^{(N)} .
$$

$I S U(1 \mid 1)$-инвариантное внутреннее произведение определяется как

$$
\langle\Phi \mid \Psi\rangle=\int d \mu \int d \xi d \bar{\xi} \bar{\Phi} \Psi=\int d \mu e^{-2 \kappa K_{2}} \int d \xi d \bar{\xi} K_{1}^{2 M} \overline{\Phi_{\mathrm{ch}}} \Psi_{\mathrm{ch}},
$$

где $d \mu=d z d \bar{z} d \zeta d \bar{\zeta}$ - мера интегрирования модели на суперплоскости. Записав разложение

$$
\Psi_{\mathrm{an}}^{(N)}=A^{(N)}(z)+\zeta B^{(N)}(z)+\xi C^{(N)}(z)+\zeta \xi B^{(N)}(z)
$$

можно показать, что

$$
\|\Psi\|^{2} \propto \int d z d \bar{z} e^{-2 \kappa|z|^{2}}\left[(2 M-N)\left(2 \kappa A^{\dagger} A+B^{\dagger} B\right)+2 \kappa C^{\dagger} C+D^{\dagger} D\right] .
$$

2 Теоретическая и математическая физика, т. 154, № 3, 2008 г. 
Таким образом, при $N>2 M>0$ и $M<0$ появляются отрицательные нормы. При $N=2 M$ присутствуют нулевые нормы.

Как и в предыдущих случаях, можно переопределить внутреннее произведение путем введения нетривиального метрического оператора на пространстве состояний. На аналитических функциях этот оператор задается выражением

$$
G_{\mathrm{an}}=\left[\xi, \partial_{\xi}\right]=-1+2 \xi \partial_{\xi}
$$

После такого переопределения квадрат нормы (под знаком интеграла) приобретает вид

$$
\propto\left[(N-2 M)\left(2 \kappa A^{\dagger} A+B^{\dagger} B\right)+2 \kappa C^{\dagger} C+D^{\dagger} D\right] .
$$

Теперь при $M<0$ все состояния обладают положительной нормой. Это справедливо и при $M=0$ с тем исключением, что половина состояний с $N=0$ имеет нулевую норму. Естественно определить (супер)пространство физических состояний как фактор по подпространству состояний с нулевыми нормами. В результате состояния с нулевыми нормами не дают вклада в физический спектр. Получаем, что при $M=0$ модель на планарном суперфлаге имеет точно такой же спектр, включая вырождение, как и модель на суперплоскости, и, следовательно, эквивалентна последней. При $M>0$ остаются отрицательные нормы для значений $N<2 M$, так что в этом интервале параметров необходимо сохранить "наивное" определение нормы.

5.2. Скрытая $\mathcal{N}=2$ суперсимметрия. Как и в модели на суперплоскости, переход к новому определению нормы меняет правило эрмитова сопряжения $I S U(1 \mid 1)$-суперзаряда $Q$, в результате чего естественным путем появляются новые сохраняющиеся суперзаряды. В применении к аналитическим волновым функциям эти суперзаряды даются выражениями

$$
S_{\mathrm{an}}=2 i \kappa \xi\left(2 M-N_{\mathrm{an}}\right), \quad S_{\mathrm{an}}^{\ddagger}=2 i \kappa \partial_{\xi}, \quad\left\{S_{\mathrm{an}}, S_{\mathrm{an}}^{\ddagger}\right\}=2 \kappa\left(H_{\mathrm{an}}-4 \kappa M\right) .
$$

Эта квантовая суперсимметрия на мировой линии существует при $M \leqslant 0$, поскольку невозможно достичь положительной определенности антикоммутатора в (5.10) при $M>0$ во всей области изменения параметров и на каждом УЛ.

При $M=0$ оба оператора $S$ и $S^{\ddagger}$ аннигилируют основное состояние $(N=0)$ по модулю состояний с нулевой нормой. В этом случае мировая суперсимметрия не нарушена, основное состояние является $\mathcal{N}=2$ синглетом и обладает только двукратным вырождением, которое связано с $I S U(1 \mid 1)$-инвариантностью. При $N>0$ волновые функции образуют нетривиальные мультиплеты $\mathcal{N}=2$ суперсимметрии, причем каждый такой мультиплет состоит из двух неприводимых $I S U(1 \mid 1)$-мультиплетов, благодаря чему возникает четырехкратное вырождение. При $M=0$ планарный суперфлаг эквивалентен модели Ландау на суперплоскости. При $M<0$ основное состояние не инвариантно относительно $\mathcal{N}=2$ суперсимметрии, которая, таким образом, спонтанно нарушена в этом случае.

Как и в модели Ландау на суперплоскости, $\mathcal{N}=2$ суперсимметрия на массовой поверхности имеет реализацию в терминах исходных $d=1$ переменных:

$$
\delta z=-\epsilon \xi(\dot{z}+\bar{\xi} \dot{\zeta}), \quad \delta \zeta=-[(1+\bar{\xi} \xi) \dot{z}+\bar{\xi} \dot{\zeta}] \bar{\epsilon}, \quad \delta \xi=-2 i \kappa \bar{\epsilon}
$$


(реализация на $\bar{z}, \bar{\zeta}, \bar{\xi}$ может быть получена путем комплексного сопряжения). С учетом уравнений движения эти преобразования на каждом поле замыкаются на производную по времени. В частности, правильное замыкание на $\xi$ получается благодаря тому, что $\dot{\xi}=0$ на массовой оболочке. Замыкание пропорционально $\kappa$, поэтому $\mathcal{N}=2$ суперсимметрия существует только при $\kappa \neq 0$, т.е., как и в модели Ландау на суперплоскости, она носит динамический характер. Было бы интересно вывести эту реализацию и действие (5.1) из подходящего $\mathcal{N}=2$ суперполевого формализма вне массовой оболочки, как это сделано в следующем разделе для модели на суперплоскости.

\section{6. ЯВНАЯ $\mathcal{N}=2$ СУПЕРСИММЕТРИЯ}

В этом разделе модель Ландау на суперплоскости (см. раздел 4) переформулирована в терминах $\mathcal{N}=2$ суперполей мировой линии.

\section{1. Суперполевое действие модели на суперплоскости.}

6.1.1. Определения. Основные объекты суть $\mathcal{N}=2, d=1$ киральные бозонное и фермионное суперполя $\Phi$ и $\Psi$ одинаковой размерности.

Вещественное $\mathcal{N}=2, d=1$ суперпространство параметризовано координатами

$$
(\tau, \theta, \bar{\theta}) .
$$

Левое и правое киральные суперпространства определяются как

$$
\left(t_{\mathrm{L}}, \theta\right), \quad\left(t_{\mathrm{R}}, \bar{\theta}\right), \quad t_{\mathrm{L}}=\tau-i \theta \bar{\theta}, \quad t_{\mathrm{R}}=\tau+i \theta \bar{\theta}=t_{\mathrm{L}}+2 i \theta \bar{\theta} .
$$

Удобно работать в левом (киральном) базисе, поэтому для краткости будем использовать обозначение $t_{\mathrm{L}} \equiv t, t_{\mathrm{R}}=t+2 i \theta \bar{\theta}$. В этом базисе $\mathcal{N}=2$ ковариантные спинорные производные имеют вид

$$
\bar{D}=-\frac{\partial}{\partial \bar{\theta}}, \quad D=\frac{\partial}{\partial \theta}-2 i \bar{\theta} \partial_{t}, \quad\{D, \bar{D}\}=2 i \partial_{t}, \quad D^{2}=\bar{D}^{2}=0 .
$$

Киральные суперполя $\Phi$ и $\Psi$ подчиняются связям

$$
\bar{D} \Phi=\bar{D} \Psi=0
$$

и в левокиральном базисе имеют следующее компонентное разложение:

$$
\Phi(t, \theta)=z(t)+\theta \chi(t), \quad \Psi(t, \theta)=\psi(t)+\theta h(t),
$$

где комплексные поля $z(t), h(t)$ представляют собой бозоны и $\chi(t), \psi(t)$ - фермионы. Разложение сопряженных суперполей в том же левокиральном базисе записывается как

$$
\bar{\Phi}=\bar{z}-\bar{\theta} \bar{\chi}+2 i \theta \bar{\theta} \dot{\bar{z}}, \quad \bar{\Psi}=\bar{\psi}+\bar{\theta} \bar{h}+2 i \theta \bar{\theta} \dot{\bar{\psi}} .
$$

Нам также потребуется компонентная структура следующих суперполей:

$$
\begin{array}{ll}
D \Phi=\chi-2 i \bar{\theta} \dot{z}+2 i \theta \bar{\theta} \dot{\chi}, & \bar{D} \bar{\Phi}=(D \Phi)^{\dagger}=\bar{\chi}+2 i \theta \dot{\bar{z}}, \\
D \Psi=h-2 i \bar{\theta} \dot{\psi}+2 i \theta \bar{\theta} \dot{h}, & \bar{D} \bar{\Psi}=-(D \Psi)^{\dagger}=-\bar{h}+2 i \theta \dot{\bar{\psi}} .
\end{array}
$$

Интеграл Березина нормирован условием

$$
\int d^{2} \theta(\theta \bar{\theta})=1
$$


6.1.2. Суперполевое действие. Суперполевое действие, приводящее в компонентах к действию модели Ландау на суперплоскости (4.1), имеет вид

$$
S=-\int d t d^{2} \theta\{\Phi \bar{\Phi}+\Psi \bar{\Psi}+\rho[\Phi D \Psi-\Phi \bar{D} \bar{\Psi}]\} \equiv \int d t d^{2} \theta\left\{\mathcal{L}_{1}+\mathcal{L}_{2}\right\} .
$$

Здесь $\rho$ - вещественный параметр. Проводя интегрирование по грассмановым переменным, получаем

$$
\begin{aligned}
& \mathcal{L}_{1} \Rightarrow-2 i(z \dot{\bar{z}}+\psi \dot{\bar{\psi}})-(\chi \bar{\chi}+h \bar{h}), \\
& \mathcal{L}_{2} \Rightarrow-2 i \rho(z \dot{h}+\chi \dot{\psi}+\dot{\bar{z}} \bar{h}+\bar{\chi} \dot{\bar{\psi}}) .
\end{aligned}
$$

Поля $h$ и $\chi$ являются вспомогательными и могут быть исключены с помощью их уравнений движения:

$$
\chi=2 i \rho \dot{\bar{\psi}}, \quad h=-2 i \rho \dot{\bar{z}} .
$$

После подстановки этих выражений в (6.10) получаем

$$
\mathcal{L} \equiv \mathcal{L}_{1}+\mathcal{L}_{2} \Rightarrow-2 i(z \dot{\bar{z}}+\psi \dot{\bar{\psi}})+4 \rho^{2}(\dot{z} \dot{\bar{z}}+\dot{\bar{\psi}} \dot{\psi}) \equiv \widetilde{\mathcal{L}}
$$

Сделав переобозначения

$$
\bar{\psi}=\zeta, \quad \psi=\bar{\zeta}, \quad 4 \rho^{2} \equiv \frac{1}{\kappa}
$$

и интегрируя по частям, лагранжиан (6.12) можно привести к виду

$$
\widehat{\mathcal{L}}=-i(z \dot{\bar{z}}-\bar{z} \dot{z}+\zeta \dot{\bar{\zeta}}-\dot{\zeta} \bar{\zeta})+\frac{1}{\kappa}(\dot{z} \dot{\bar{z}}+\dot{\zeta} \dot{\bar{\zeta}})
$$

Он совпадает с лагранжианом модели на суперплоскости (4.1) с точностью до обращения времени $t \rightarrow-t$ и общего коэффициента $-\kappa$ перед действием.

\section{2. Симметрии.}

6.2.1. Суперсимметрия. По построению суперполевое действие (6.9) обладает явной $\mathcal{N}=2$ суперсимметрией. $\mathcal{N}=2$ преобразования компонентных полей могут быть найдены из суперполевых вариаций

$$
\delta \Phi=-[\epsilon Q-\bar{\epsilon} \bar{Q}] \Phi, \quad \delta \Psi=-[\epsilon Q-\bar{\epsilon} \bar{Q}] \Psi
$$

где в левокиральном базисе

$$
Q=\frac{\partial}{\partial \theta}, \quad \bar{Q}=-\frac{\partial}{\partial \bar{\theta}}-2 i \theta \partial_{t}, \quad\{Q, \bar{Q}\}=-2 i \partial_{t}=2 P_{0} .
$$

Из (6.15), (6.16) следует, что вне массовой оболочки

$$
\delta z=-\epsilon \chi, \quad \delta \chi=2 i \bar{\epsilon} \dot{z}, \quad \delta \psi=-\epsilon h, \quad \delta h=2 i \bar{\epsilon} \dot{\psi} .
$$

После подстановки выражений (6.11) для вспомогательных полей и с учетом переобозначений (6.13) эти преобразования принимают вид

$$
\delta z=-\frac{i}{\sqrt{\kappa}} \epsilon \dot{\zeta}, \quad \delta \zeta=-\frac{i}{\sqrt{\kappa}} \bar{\epsilon} \dot{z} .
$$

Они совпадают с (4.15) (с точностью до перенормировки параметров $\epsilon, \bar{\epsilon}$ ). 
6.2.2. Две $I S U(1 \mid 1)$-симметрии. Суперполевые $I S U(1 \mid 1)$-преобразования имеют несколько более сложный вид. Главное требование к ним - совместность с условиями киральности (6.4). В то время как супертрансляции в пространстве отображения действуют как сдвиги $\Phi$ и $\Psi$ :

$$
\delta_{\mathrm{b}} \Phi=b, \quad \delta_{\beta} \Psi=\nu,
$$

где $b$ и $\nu$ - комплексные константные бозонные и фермионные параметры, нечетные $S U(1 \mid 1)$-преобразования вне массовой оболочки содержат в явном виде $\theta$ и $\bar{\theta}$ :

$$
\begin{array}{ll}
\delta \Phi=\bar{D}\left(\omega \bar{\theta} \bar{\Psi}-\frac{1}{2 \sqrt{\kappa}} \bar{\omega} \theta D \Phi\right), & \delta \Psi=\bar{D}\left(\omega \bar{\theta} \bar{\Phi}-\frac{1}{2 \sqrt{\kappa}} \bar{\omega} \theta D \Psi\right), \\
\delta \bar{\Phi}=D\left(\bar{\omega} \theta \Psi-\frac{1}{2 \sqrt{\kappa}} \omega \bar{\theta} \bar{D} \bar{\Phi}\right), & \delta \bar{\Psi}=-D\left(\bar{\omega} \theta \Phi+\frac{1}{2 \sqrt{\kappa}} \omega \bar{\theta} \bar{D} \bar{\Psi}\right),
\end{array}
$$

где $\omega, \bar{\omega}$ - соответствующие грассмановы параметры преобразований. Эти преобразования согласованы с киральностью и антикиральностью суперполевых вариаций благодаря присутствию проекторов $\bar{D}$ и $D$ в их правых частях. Преобразования компонентных полей имеют вид

$$
\delta z=\omega \bar{\psi}, \quad \delta \chi=\frac{i}{\sqrt{\kappa}} \bar{\omega} \dot{z}, \quad \delta \psi=\omega \bar{z}, \quad \delta h=\frac{i}{\sqrt{\kappa}} \bar{\omega} \dot{\psi}
$$

(надо добавить и комплексно-сопряженные к ним). Они замыкаются на $C$-преобразования, реализованные как

$$
\delta_{\mathrm{c}} z=i \alpha z, \quad \delta_{\mathrm{c}} \psi=-i \alpha \psi, \quad \delta_{\mathrm{c}} \chi=\frac{i}{\sqrt{\kappa}} \alpha \dot{\bar{\psi}}, \quad \delta_{\mathrm{c}} h=\frac{i}{\sqrt{\kappa}} \alpha \dot{\bar{z}}
$$

(плюс комплексно-сопряженные вариации), где $\alpha$ - параметр преобразования. Выражения для вспомогательных полей на массовой оболочке (6.9) совместимы с преобразованиями (6.21), (6.22). Имеется полное соответствие между преобразованиями (6.21), (6.22) и $I S U(1 \mid 1)$-генераторами (4.3).

Из-за явного присутствия $\theta$ в (6.20) эти $S U(1 \mid 1)$-преобразования не коммутируют с $\mathcal{N}=2$ суперсимметрией мировой линии. Любопытно, что существует и другой тип "внутренних" нечетных преобразований, которые по построению коммутируют с $\mathcal{N}=2$ суперсимметрией:

$$
\delta \Phi=\lambda\left(\Psi+\frac{1}{2 \sqrt{\kappa}} \bar{D} \bar{\Phi}\right), \quad \delta \Psi=\bar{\lambda}\left(\Phi-\frac{1}{2 \sqrt{\kappa}} \bar{D} \bar{\Psi}\right),
$$

где $\lambda$ - соответствующий грассманов параметр. На компонентных полях вариации (6.23) порождают следующие переобразования:

$$
\begin{aligned}
& \delta z=\lambda\left(\psi+\frac{1}{2 \sqrt{\kappa}} \bar{\chi}\right) \Rightarrow \lambda\left(\psi-\frac{i}{2 \kappa} \dot{\psi}\right), \\
& \delta \psi=\bar{\lambda}\left(z+\frac{1}{2 \sqrt{\kappa}} \bar{h}\right) \Rightarrow \bar{\lambda}\left(z+\frac{i}{2 \kappa} \dot{z}\right), \\
& \delta \chi=-\lambda\left(h+\frac{i}{\sqrt{\kappa}} \dot{\bar{z}}\right) \Rightarrow 0, \\
& \delta h=-\bar{\lambda}\left(\chi-\frac{i}{\sqrt{\kappa}} \dot{\bar{\psi}}\right) \Rightarrow 0 .
\end{aligned}
$$


Здесь стрелка означает переход к вариации на массовой поверхности (со вспомогательными полями, исключенными в соответствии с (6.11)). Легко проверить инвариантность (6.12) и (6.14) относительно (6.24), (6.25). Преобразования (6.23) порождают $S U(1 \mid 1)$-супергруппу, отличающуюся от “стандартной” (6.20)-(6.22). Вычисляя скобку Ли двух преобразований (6.26), находим явную суперполевую реализацию соответствующего $\widetilde{C}$-преобразования:

$$
\delta_{\tilde{c}} \Phi=i \beta\left(\Phi+\frac{i}{2 \kappa} \dot{\Phi}\right), \quad \delta_{\tilde{c}} \Psi=i \beta\left(\Psi+\frac{1}{\sqrt{\kappa}} \bar{D} \bar{\Phi}+\frac{i}{2 \kappa} \dot{\Psi}\right) .
$$

Как и следовало ожидать, эти преобразования коммутируют с (6.23). Вместе с супертрансляциями (6.19) $S U(1 \mid 1)$-преобразования (6.23) и (6.27) образуют еще одну супергруппу $I S U(1 \mid 1)$. Эта супергруппа не коммутирует со “стандартной” (хотя обе имеют общий супертрансляционный сектор): замыкание этих двух супергрупп содержит некоторые новые преобразования, которые, по-видимому, порождают бесконечномерную группу симплектических диффеоморфизмов суперпространства отображения (присутствие скрытых симметрий такого типа в действии (6.12) на массовой поверхности отмечено в работе [7]).

Остается построить $\mathcal{N}=2$ суперполевую формулировку модели планарного суперфлага. Ее основными элементами должны быть те же суперполя $\Phi$ и $\Psi$, дополненные новым голдстоуновским киральным спинорным суперполем $\Omega=\xi(t)+\theta g(t)$. Формулировка компонентного действия (5.1) в формализме первого порядка [7] может стать подходящей отправной точкой для такого построения. Одной из интересных проблем для дальнейших исследований является построение моделей, подобных модели на суперфлаге, исходя из $\mathcal{N}=2$ суперполевого формализма. Отметим также, что подход, изложенный в этом разделе, по-видимому, может быть использован для построения обобщений моделей на суперплоскости и суперфлаге со скрытой $\mathcal{N}=4$ суперсимметрией на мировой линии (и, возможно, суперсимметриями c $\mathcal{N}>4)$.

\section{7. РЕЗЮМЕ}

Кратко суммируем содержание работы.

Самосогласованные суперрасширения бозонной модели Ландау существуют [4]-[9] и могут интерпретироваться как сигма-модели ВЗВ-типа на соответствующих градуированных расширениях группы "магнитных трансляций", лежащей в основе исходной модели Ландау.

Несмотря на появление нежелательных отрицательных норм при "естественном" выборе инвариантного внутреннего произведения эту трудность можно обойти, переопределив внутреннее произведение в духе работ [10], [11].

Интригующей общей чертой планарных суперрасширений модели Ландау является наличие скрытой динамической $\mathcal{N}=2$ суперсимметрии [8], [9]. Сохраняется ли это свойство в моделях Ландау на суперсфере и суперфлаге? Возможно ли во всех случаях воспроизвести его исходя из альтернативного суперполевого формализма, как в модели на суперплоскости? Существуют ли обобщения на высшие $\mathcal{N}$ ? Желательно иметь ответы на эти вопросы. 
Что касается физических применений, то прежде всего хотелось бы полностью осознать, какие явления описываются суперсимметричными версиями КЭХ и каков физический смысл дополнительных фермионных переменных.

Благодарности. Я благодарен организаторам семинара "Классические и квантовые интегрируемые системы”, пригласившим меня сделать доклад, ставший основой этой статьи. Большинство представленных результатов получено в сотрудничестве с Т. Картрайтом, Л. Мезинческу и П. К. Таунсендом, которым я выражаю свою глубокую признательность. Работа поддержана РФФИ (грант № 06-02-16684), INTAS (грант № 05-7928) и Программой Гейзенберг-Ландау. Я благодарен Laboratoire de Physique Ecole Normale Supérieure (Lyon, France) за гостеприимство на последних этапах этой работы и $\Phi$. Дельдуку за полезные замечания.

\section{Список литературы}

[1] L. Landau, Z. Phys., 64 (1930), 629-637.

[2] F. D. M. Haldane, Phys. Rev. Lett., 51 (1983), 605-608.

[3] Z.F. Ezawa, Quantum Hall Effects. Field Theoretical Approach and Related Topics, World Scientific, Singapore, 2000.

[4] E. Ivanov, L. Mezincescu, P. K. Townsend, Fuzzy $C P^{(n \mid m)}$ as a quantum superspace, arXiv: hep-th/0311159.

[5] E. Ivanov, L. Mezincescu, P.K. Townsend, A super-flag Landau model, arXiv: hep-th/0404108.

[6] K. Hasebe, Y. Kimura, Nucl. Phys. B, 709 (2005), 94-114; arXiv: hep-th/0409230; K. Hasebe, Phys. Rev. Lett., 94 (2005), 206802; arXiv: hep-th/0411137.

[7] E. Ivanov, L. Mezincescu, P. K. Townsend, JHEP, 01 (2006), 143; arXiv: hep-th/0510019.

[8] T. Curtright, E. Ivanov, L. Mezincescu, P. K. Townsend, JHEP, 04 (2007), 020; arXiv: hep-th/0612300.

[9] K. Hasebe, Phys. Rev. D, 72 (2005), 105017; arXiv: hep-th/0503162.

[10] C. M. Bender, Contemp. Phys., 46 (2005), 277-292; arXiv: quant-ph/0501052; Making sense of non-Hermitian Hamiltonians, arXiv: hep-th/0703096.

[11] T. Curtright, L. Mezincescu, Biorthogonal quantum systems, arXiv: quant-ph/0507015.

[12] H. Elvang, J. Polchinski, The Quantum Hall effect on $R^{4}$, arXiv: hep-th/0209104.

[13] J. Madore, Classical Quantum Gravity, 9 (1992), 69-87.

[14] E. Witten, Nucl. Phys. B, 188:3 (1981), 513-554.

[15] В. П. Акулов, А. И. Пашнев, ТМФ, 65 (1985), 84-92.

[16] Д. В. Волков, А. И. Пашнев, ТМФ, 44:3 (1980), 321-326.

[17] D. Robert, A. V. Smilga, Supersymmetry vs ghosts, arXiv: math-ph/0611023. 